BÚSQUEDA - Julio / Diciembre de 2015 - No. 15 (18 - 29)

\title{
Protectores ante la violencia escolar en contexto dominicano: Un modelo explicativo desde la psicología positiva
}

\author{
Amparo Oliver Germes ${ }^{1}$, Lesia María Bautista Gómez², Laura Galiana Llinares ${ }^{1}$ Ana María Descalzo \\ Viñals', Elena Terreros García1', Varinia Bustos Álvarez ${ }^{3}$ \\ Recibido: 6-10-2015 - Aceptado: 19-11-2015
}

\begin{abstract}
Resumen
Determinados comportamientos agresivos de los adolescentes preocupan en escuelas de todo el mundo, siendo un problema de especial relevancia en América Latina y región Caribe. En concreto, en la República Dominicana los estudios hablan de prevalencias del 21.8 al 33.6\%. El objetivo de este estudio es conocer en qué medida variables como el autoconcepto, la esperanza disposicional, las actitudes emprendedoras y los valores pueden actuar como factores protectores frente a la ocurrencia de dichas conductas violentas. Para ello, se llevó a cabo un estudio en una muestra de 117 estudiantes dominicanos de secundaria. La evaluación incluyó, además de los datos socio-demográficos, escalas de medida de conductas violentas en la escuela, esperanza disposicional, autoconcepto forma 5, actitudes emprendedoras para estudiantes y valores personales. Para evaluar la relación entre las diversas dimensiones, se planteó y estimó un modelo de ecuaciones estructurales MIMIC (multiple indicators multiple causes). Bajo este modelo, la esperanza, las actitudes emprendedoras, los valores personales y cuatro dimensiones del autoconcepto predicen con buen ajuste la conducta violenta en la escuela, correctamente aproximada a través de un modelo de medida en seis dimensiones. Los resultados del modelo ofrecen información útil desde dos perspectivas: a) Acercamiento empírico al constructo violencia escolar con saturaciones factoriales satisfactorias para los indicadores de la variable latente o factor; $b$ ) Identificación de variables predictoras de la violencia: esperanza, empatía, toma de riesgos en contexto de emprendimiento, valores mixtos (combinación de individual y colectivo) y autoconcepto familiar. En la discusión se recoge la necesidad de trabajar desde la Psicología Positiva las variables halladas más relevantes, para disminuir las conductas violentas en las escuelas de la República Dominicana.
\end{abstract}

Palabras clave: Violencia escolar; autoconcepto; esperanza disposicional; estudiantes; modelo de ecuaciones estructurales; factores protectores.

\footnotetext{
${ }^{1}$ Universidad de Valencia, España.

${ }^{2}$ Liceo Técnico P. Henríquez e INFOTEP. Universidad Autónoma de Santo Domingo. República Dominicana.

${ }^{3}$ Universidad ESAN, Perú.
} 
Amparo Oliver G., et al., - Protectores ante la violencia escolar

\title{
Guards to the dominican school violence in context: An explanatory model from positive psychology
}

\begin{abstract}
Some aggressive behaviors of adolescents in schools became a particularly important problem in Latin American and Caribbean region. Specifically, in the Dominican Republic studies report of prevalence from 21.8 to $33.6 \%$. The aim of this research is to study if some variables from positive psychology such as self-concept, dispositional hope, entrepreneurial attitudes, and values can act as protective factors for such violent behaviors. In order to reach our goal, a study on a sample of 117 secondary students was carried out. Assessment tool kit included, together with information on socio-demographic data, the Violent Behaviors on School Scale, the Dispositional Hope Scale, the Self-Concept 5 Scale, the Entrepreneurial Attitudes Scale for Students, and the Personal Values Questionnaire. To evaluate the relation among the different dimensions, a MIMIC (multiple indicators multiple causes) structure equation model was estimated. Under this good fitting model, hope, entrepreneurial attitudes, personal values and four dimensions of self-concept successfully predict violent behavior in school approached through a rough measurement model based on six dimensions. The model results could offer useful information from two perspectives: a) Empirically approaching to the construct school violence with satisfactory factor loadings in this latent variable; b) Identifying predictors of violence, as hope, empathy, risk-taking in context of entrepreneurship, mixed values (combination of individual and collective ones) and family selfconcept. Discussion section encourages the need to work through positive psychology variables trying to decrease the presence of violent behavior in schools in the Dominican Republic.
\end{abstract}

Key words: School violence; self-concept; hope; students; structural equation modeling; protective factors.

\section{Introducción}

Durante los últimos años, el comportamiento agresivo de los adolescentes dentro de las escuelas de todo el mundo, ha recibido creciente interés en la sociedad y comunidad científica. Desde cuando Dan Olweus, pionero en el estudio de la violencia escolar empezó sus investigaciones en la década de 1970, han proliferado las investigaciones acerca de este tema. Pese a esta realidad, la literatura científica existente a este respecto en Latinoamérica y Caribe es todavía escasa. De acuerdo con informes del año 2000, emitidos a través del Banco Interamericano de Desarrollo y el Banco Mundial, América Latina sería el continente más violento y el que más dinero invierte para combatir la violencia social (Quintana, Montgomery y Malaver, 2009). La violencia juvenil, es en este contexto una de las problemáticas más recurrentes, conformada por un amplio abanico de actos agresivos que van desde la intimidación y las peleas hasta formas más graves de agresión y homicidio (OMS, 2002). Un estudio llevado a cabo por la Comisión Económica para América Latina y Caribe (CEPAL, 2011) situó a la República Dominicana en tercer lugar entre los cinco países de América Latina con mayor índice de violencia física entre pares. En primer lugar se situó Argentina con un 23.5\%; después Ecuador con $21.9 \%$, República Dominicana con $21.8 \%$; y Costa Rica y Nicaragua ambas con un 21.2\% (Román y Murillo, 2011). En concreto, en el "Estudio de prevalencia, tipología y causas de la violencia en los centros educativos de básica y media de la República Dominicana", que abarcó a estudiantes, maestros, directores y padres, se señala recientemente que la prevalencia del acoso escolar es alta en este país, con una incidencia de $33.6 \%$ en las escuelas públicas (Vargas, 2014). Los estudiantes informaron que el acoso verbal es el más común, y ocurre con mayor frecuencia durante los momentos de ocio, con un $51.6 \%$, seguido del que se vive en las aulas, con el docente presente, con un $41.2 \%$. 
BÚSQUEDA - Julio / Diciembre de 2015 - No. 15 (18 - 29)

Esta manifestación de comportamiento agresivo se refiere a cualquier tipo de conducta violenta en contextos escolares, dirigida a hacer daño a alumnos, profesores o propiedades, de manera ocasional o repetida con cierta frecuencia (Serrano e Iborra, 2005). Estudios todavía más actuales delimitan la violencia escolar en torno a dos categorías, tipo y función de la agresión (Moreno, Estévez, Murgui y Musitu, 2009; Moreno, Ramos, Martínez y Musitu, 2010).

El tipo de agresión puede clasificarse como manifiesta o relacional. La primera ha sido la más visible y estudiada durante los últimos años, y se refiere a una confrontación directa hacia otros, ya sea física o verbal, con la intención de causar daño. Por otro lado, la agresión relacional, aunque más sutil y menos visible, es igual de dañina que la agresión manifiesta, pero no implica una confrontación directa entre la víctima y el agresor, sino que está dirigida a provocar daño en el círculo de amistad o percepción de pertenencia a un grupo, lo que afecta al bienestar psicosocial de sus víctimas.

La función de la agresión, por su parte, puede clasificarse como defensiva (reactiva) u ofensiva (pura o instrumental). La agresión reactiva suele estar relacionada con una baja resistencia a la frustración y hace referencia a comportamientos que suponen una respuesta defensiva ante alguna provocación percibida o real. La agresión ofensiva puede ser pura o instrumental. La pura se desencadena sin la intervención de un estímulo agresivo, mientras que la instrumental consiste en actos intencionales planificados y premeditados de violencia como medio para resolver conflictos, controlar el comportamiento de los demás o conseguir beneficios o recompensas (Velasco, 2013). Es este último tipo de agresión el más relacionado con futuros problemas de delincuencia, pero también con altos niveles de liderazgo y competencia social.

Para hacer frente a esta violencia, diversos factores se han señalado como protectores. Uno de los constructos sobre los que se ha aportado evidencia es el de autoconcepto. Diversos estudios han establecido una relación entre aspectos relativos al ajuste emocional, como un bajo autoconcepto, relacionándolo con conductas violentas en la adolescencia (Martínez, Buelga y Cava, 2007; Moreno et al., 2009). La capacidad de los estudiantes para establecer sus guías y metas podría actuar también como factor protector frente a la violencia escolar. Esta esperanza disposicional o Hope en la literatura anglosajona, se relaciona, a su vez, con el emprendimiento o las actitudes emprendedoras. El autoconcepto se ha relacionado recientemente con la esperanza disposicional, definida como constructo motivacional (Snyder et al., 1991). En este sentido, la capacidad de los estudiantes para establecer sus guías y metas podría actuar también como factor protector frente a la violencia escolar. Pensemos que cualquier proceso de empoderamiento tiene implicaciones a nivel individual, organizacional, político, sociológico, económico y espiritual (Soledad, Hopkins y Mirna, 2007). También, que la educación formal hacia el autoempleo en la universidad facilita el proceso de creación de empresas porque permite concienciar a los estudiantes de la viabilidad que tiene el auto-empleo como salida profesional (Vesper, 1990), pero también incrementa los valores y el autoconcepto de los estudiantes. De esta forma, quien emprende desea tener el control de las situaciones, presenta mayor necesidad de autonomía y concede importancia a la individualidad, pero también a la libertad (Kirby, 2004).

En efecto, factores como el autoconcepto, la esperanza, los valores o las actitudes emprendedoras podrían actuar como factores protectores frente a las conductas violentas. Estas variables identificadas como protectoras, están directamente relacionadas con el objeto de estudio de la Psicología positiva, pues esta se ocupa de investigar acerca de las fortalezas y virtudes humanas y los efectos que estas tienen en las vidas de las personas y en las sociedades en que viven (Cuadra y Florenzano, 2003 citado en Sánchez et. al 2010). Utilizando una estrategia de investigación cuantitativa, de corte transversal, el objetivo de este estudio es poner a prueba estas hipótesis de forma conjunta a través de un modelo explicativo en una muestra de estudiantes de la República Dominicana. 


\section{Metodología}

\section{Diseño, procedimiento y muestra}

La muestra estuvo constituida por 117 estudiantes de ambos sexos (51 chicos y 66 chicas) de edades comprendidas entre 16 y 19 años ( promedio $=17.66$; desviación típica $=.790$ ); pertenecientes a un centro de enseñanza de la región de San Juan de la Maguana (República Dominicana). Este centro fue elegido por ser el mayor en número de alumnos y de estudios ofertados, representativo de un área geográfica que reúne condiciones como su proximidad a Haití y una tradicional escasez de recursos socioeconómicos.

Durante el pilotaje del estudio no se detectaron problemas de comprensión ni dificultades atribuibles a ítems de escalas en un español que pudiera tener matices lingüísticos diferentes a los propios del contexto dominicano. La recogida de datos se llevó a cabo en un único momento temporal, tratándose de un estudio de tipo transversal y correlacional. Los alumnos completaron los distintos cuestionarios en las aulas, con una duración aproximada de 30 minutos. En todo caso estuvieron presentes los investigadores 0 , en su defecto, personal formado para tales efectos.

\section{Instrumentos}

Además de evaluar datos socio-demográficos, la encuesta estuvo compuesta de los siguientes instrumentos:

- Escala de Conductas Violentas en la Escuela (Little, Henrich, Jones y Hawley, 2003). Esta escala está formada por 25 ítems y va dirigida a adolescentes/jóvenes (mayores de 11 años). Los ítems evalúan, con un rango de respuesta de 1 a 4 (nunca, pocas veces, muchas veces, y siempre), dos tipos de conducta violenta en el contexto escolar: por un lado, la agresión manifiesta o directa, en sus formas pura, reactiva e instrumental; por otro lado, la agresión relacional o indirecta, también en sus formas pura, reactiva e instrumental. En estudios previos realizados con otras muestras de adolescentes españoles se han obtenido adecuados índices de fiabilidad que oscilan entre .62 y .84 (Cava, Musitu y Murgui, 2006; Estévez, Martínez, Moreno y Musitu, 2006; Estévez, Martínez y Musitu, 2006; Jiménez, Moreno, Murgui y Musitu, 2008; Musitu, Estévez y Emler, 2007). En este estudio, la fiabilidad fue de .96.

- Escala de Esperanza Disposicional (Snyder et al., 1991). Se trata de un instrumento de 8 ítems desarrollado para medir esperanza, con 4 ítems para el factor vías (pathways), evaluando habilidades para identificar formas factibles de conseguir objetivos, y 4 ítems en el factor acción (agency), que miden motivaciones para perseguir objetivos. Los ítems puntúan desde 1 (definitivamente falso) a 4 (definitivamente verdadero). Se ha utilizado la versión traducida al español (Galiana, Oliver, Sancho y Tomás, 2015). El alfa en este estudio fue de .70.

- Escala autoconcepto forma 5 (AF5; García y Musitu, 1999; Bustos, Oliver y Galiana, en prensa). Esta escala está compuesta por 30 ítems y evalúa 5 dimensiones del autoconcepto: académico, familiar, físico, social y emocional, con seis ítems cada una. Va dirigida a jóvenes de 12 a 20 años. Los ítems puntúan en una escala tipo Likert que admite 11 posibilidades de respuesta $(0=$ nunca y $10=$ siempre). Se han utilizado las dimensiones académica, familiar, física y social. El alfa de Cronbach en esta muestra fue de .74.

- Escala de actitudes emprendedoras para estudiantes (Oliver y Galiana, 2015). Se trata de una escala que evalúa los principales rasgos de la personalidad emprendedora mediante 18 ítems: proactividad, filosofía o ética profesional, empatía, innovación, autonomía y toma de riesgos. Cada dimensión se evalúa mediante 3 ítems que puntúan de 1 ("muy en desacuerdo") a 7 ("muy de acuerdo"). En esta muestra, el alfa tuvo un valor de .92 .

- Cuestionario de Valores Personales (Schwartz y Bilsky, 1990). Escala con 34 valores de los recogidos por Schwartz y Bilsky que se organizan en tres factores que responden a agrupaciones de valores catalogados como individualistas, otros como colectivos y otros como mixtos en el caso de que confluyen aspectos de ambas 
BÚSQUEDA - Julio / Diciembre de 2015 - No. 15 (18 - 29)

perspectivas. El cuestionario evalúa la importancia que tiene cada valor como principio que guía la vida de la persona encuestada, usando para ello una escala tipo Likert de cinco puntos, desde 0 (nada importante en mi vida) hasta 7 (de mucha importancia en mi vida). La fiabilidad de la escala en este estudio fue de .95 .

\section{Análisis estadísticos}

Para analizar las relaciones de la conducta violenta con las distintas variables predictoras, se estimó y puso a prueba un modelo de ecuaciones estructurales, en concreto, un modelo MIMIC (multiple indicators multiple causes), con correcciones robustas para los errores estándar. Los modelos MIMIC ofrecen un contexto de error libre de medida en la variable dependiente. En el modelo propuesto, la esperanza, las variables de emprendimiento, los valores personales y cuatro dimensiones del autoconcepto, todas ellas variables observables, predecían la conducta violenta en la escuela, que a su vez explicaba las 6 dimensiones de esta clase de conducta que recoge la escala utilizada (agresión manifiesta pura, agresión manifiesta reactiva, agresión manifiesta instrumental, agresión relacional pura, agresión relacional reactiva, agresión relacional instrumental). Se estimaron las correlaciones entre las diferentes variables exógenas (independientes) del modelo. El modelo concreto a evaluar se puede observar en la figura 1.

El ajuste del modelo se evaluó utilizando diversos criterios, tal y como recomienda la literatura (Hu y Bentler, 1999; Tanaka, 1993): (a) $\chi^{2}$ (Kline, 2011); (b) Índice de bondad de ajuste comparativo (Comparative Fit Index, CFI), basado en el parámetro de no-centralidad, de más de .90 (idealmente más de .95; Hu y Bentler, 1999); y (c) Raíz del residuo cuadrático promedio de aproximación (Root Mean Square Error of Approximation, RMSEA) de .05 o menos.

Figura 1. Modelo de ecuaciones estructurales a evaluar

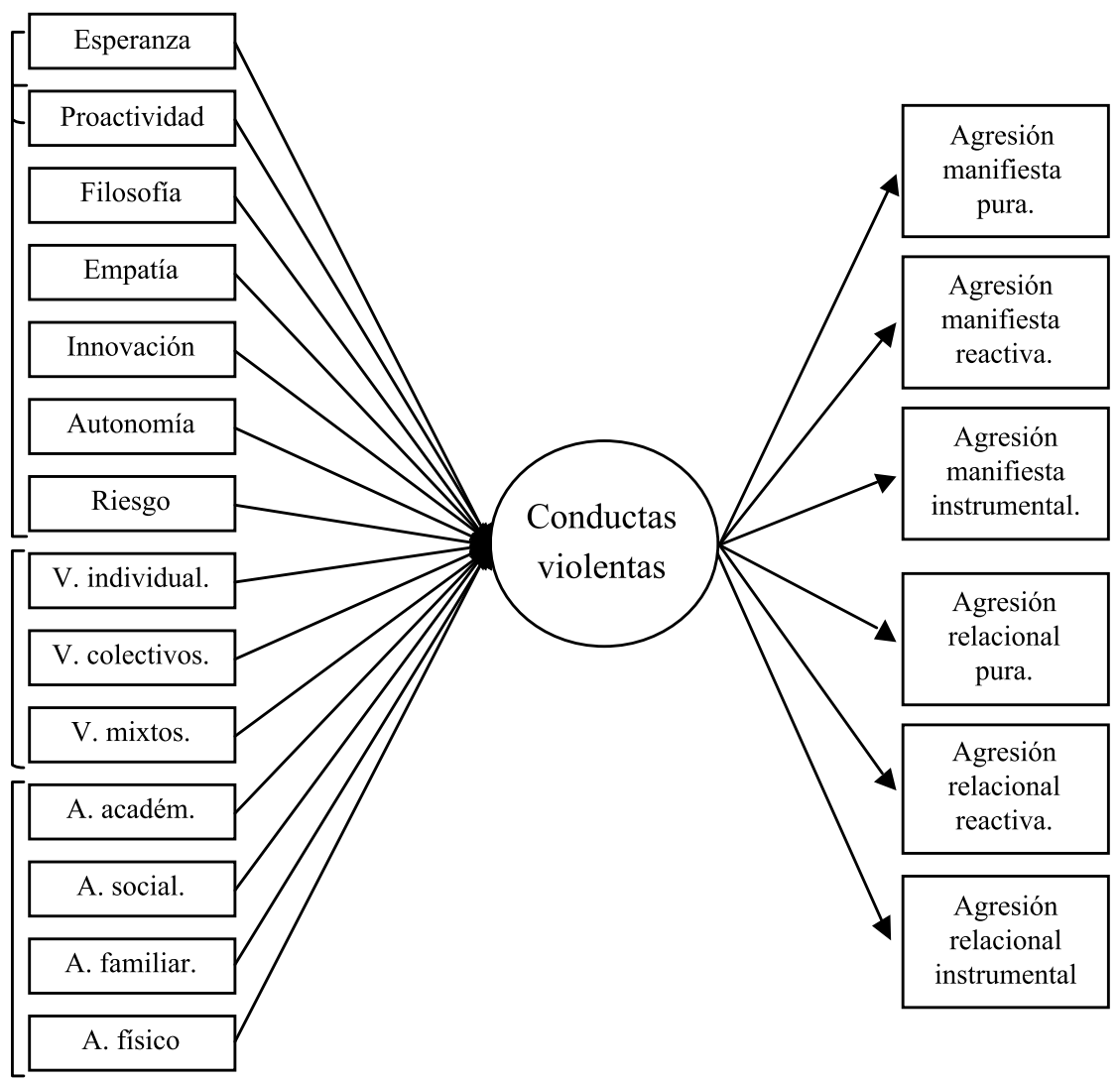

Nota: Para mayor claridad, no se muestran los errores estándar ni las correlaciones entre las variables exógenas. 


\section{Resultados}

El modelo MIMIC para la predicción de la conducta violenta en la escuela ofreció unos índices de ajuste adecuados: $\chi^{2}(79)=217.54$, $\mathrm{p}<.01 ; \mathrm{CFI}=.954 ; \mathrm{y}$ RMSEA $=.123$ (intervalo confianza del $90 \%=.103-.142$ ). El ajuste analítico también fue adecuado, tal y como se puede observar en la figura 2.
Por lo que respecta a la parte de la estructura de la conducta violenta, se realizó un examen de las cargas factoriales, lo que ofrece información complementaria sobre el ajuste analítico del modelo. Todos los indicadores cargan de forma significativa $(p<.05)$ en la dimensión hipotetizada, con saturaciones estandarizadas altas, entre un mínimo de .85 para la agresión manifiesta pura y un máximo de .94 para la agresión manifiesta

Figura 2. Parámetros estandarizados del modelo de ecuaciones estructurales

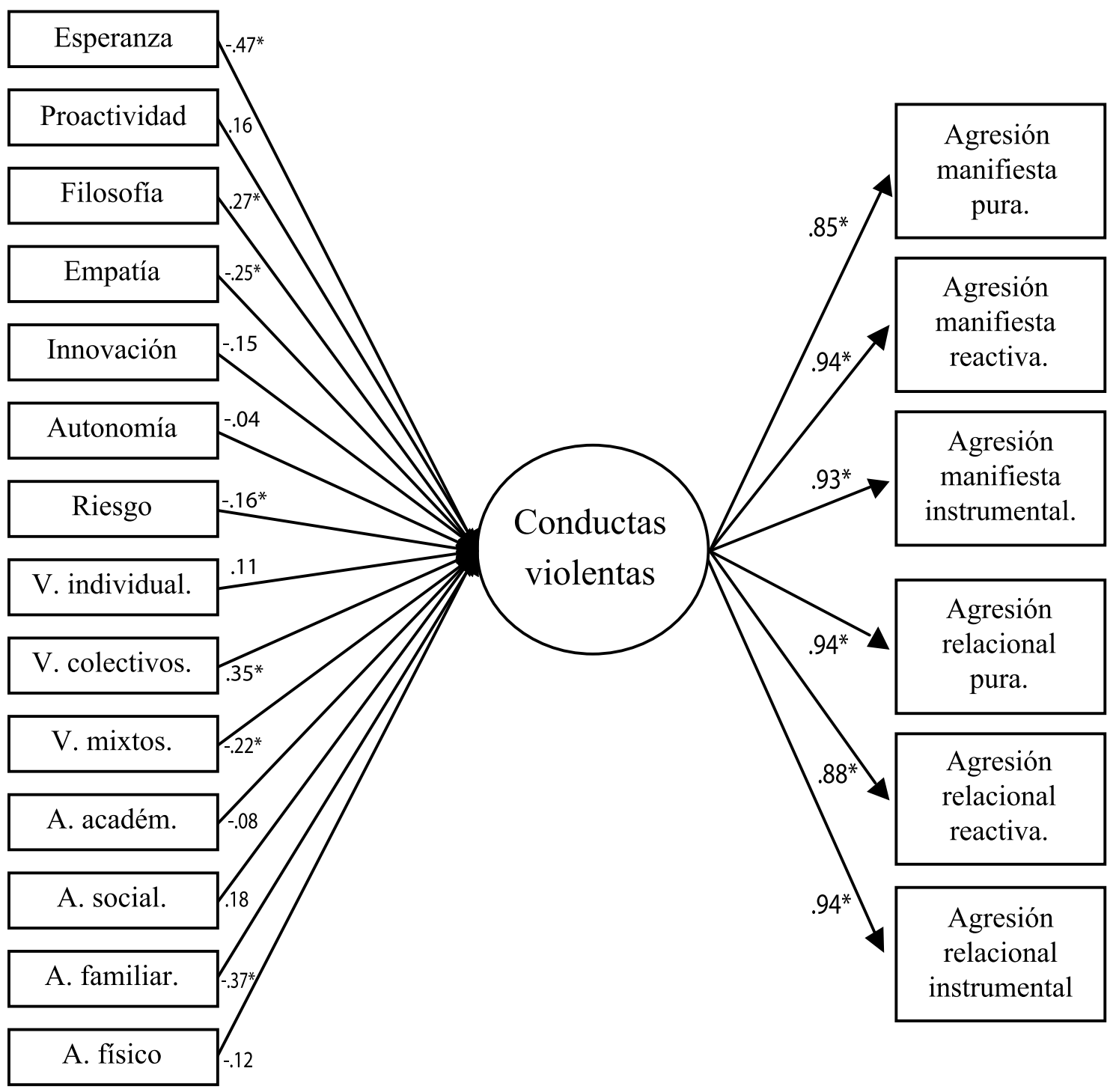

Nota: * $p<.05$. Para mayor claridad, no se muestran los errores estándar. Las correlaciones entre las variables exógenas se muestran en la Tabla 1. 
BÚSQUEDA - Julio / Diciembre de 2015 - No. 15 (18 - 29)

reactiva, agresión relacional pura y agresión relacional instrumental.

En lo que se refiere a la parte predictiva del modelo, se observaron siete predictores estadísticamente significativos: esperanza, filosofía de trabajo, empatía, toma de riesgos, valores colectivos, autoestima social y autoestima familiar. La esperanza, empatía, toma de riesgos y autoconcepto familiar predijeron la conducta violenta en negativo, es decir, altas puntuaciones en estas variables se asociaron con menores puntuaciones en conducta violenta. Filosofía de trabajo, valores colectivos y autoestima social, por su parte, se relacionaron con la conducta violenta de forma positiva, de manera que mayores puntuaciones en estas tres variables se asociaron con mayor conducta violenta.

Finalmente, las correlaciones entre las variables predictoras del modelo se muestran en la tabla 1. Tal y como se puede observar, casi todas las correlaciones fueron estadísticamente significativas y positivas, a excepción de las correlaciones de filosofía de trabajo con proactividad, autoconcepto social y autoconcepto físico; y las correlaciones de toma de riesgos con empatía, autoconcepto familiar y físico.

Tabla 1. Correlaciones entre las variables exógenas del modelo de ecuaciones estructurales

\begin{tabular}{|c|c|c|c|c|c|c|c|c|c|c|c|c|c|}
\hline & 1 & 2 & 3 & 4 & 5 & 6 & 7 & 8 & 9 & 10 & 11 & 12 & 13 \\
\hline $1 \mathrm{ESP}$ & 1.00 & & & & & & & & & & & & \\
\hline 2 PRO & $.48^{*}$ & 1.00 & & & & & & & & & & & \\
\hline $3 \mathrm{FIL}$ & .05 & $.35^{*}$ & 1.00 & & & & & & & & & & \\
\hline 4 EMP & $.31^{*}$ & $.55^{\star}$ & $.48^{\star}$ & 1.00 & & & & & & & & & \\
\hline 5 INN & $.43^{*}$ & $.74^{*}$ & $.47^{\star}$ & $.72^{*}$ & 1.00 & & & & & & & & \\
\hline 6 AUT & $.57^{\star}$ & $.66^{\star}$ & $.33^{\star}$ & $.40^{\star}$ & $.55^{\star}$ & 1.00 & & & & & & & \\
\hline 7 RIESG & $.38^{\star}$ & $.44^{*}$ & $.22^{*}$ & .13 & $.26^{\star}$ & $.69^{*}$ & 1.00 & & & & & & \\
\hline 8 IND & $.64^{*}$ & $.52^{*}$ & $.16^{\star}$ & $.48^{*}$ & $.51^{\star}$ & $.65^{\star}$ & $.45^{\star}$ & 1.00 & & & & & \\
\hline $9 \mathrm{COL}$ & $.70^{\star}$ & $.29^{*}$ & $-.14^{*}$ & .16 & $.20^{\star}$ & $.55^{\star}$ & $.35^{\star}$ & $.77^{\star}$ & 1.00 & & & & \\
\hline 10 MIXT & $.68^{\star}$ & $.45^{\star}$ & -.01 & $.26^{*}$ & $.43^{\star}$ & $.62^{*}$ & $.35^{\star}$ & $.85^{\star}$ & $.86^{\star}$ & 1.00 & & & \\
\hline 11 ACAD & $.71^{*}$ & $.46^{*}$ & .15 & $.46^{*}$ & $.52^{\star}$ & $.50^{\star}$ & $.23^{*}$ & $.55^{\star}$ & $.51^{*}$ & $.57^{\star}$ & 1.00 & & \\
\hline $12 \mathrm{SOC}$ & $.60^{\star}$ & $.28^{*}$ & -.02 & $.25^{\star}$ & $.26^{\star}$ & $.49^{*}$ & $.22^{*}$ & $.47^{\star}$ & $.52^{\star}$ & $.47^{\star}$ & $.54^{*}$ & 1.00 & \\
\hline 13 FAM & $.64^{*}$ & $.37^{\star}$ & $.17^{\star}$ & $.47^{\star}$ & $.50^{\star}$ & $.43^{*}$ & .10 & $.58^{\star}$ & $.53^{\star}$ & $.59^{\star}$ & $.69^{*}$ & $.68^{*}$ & 1.00 \\
\hline 14 FÍSIC & $.55^{\star}$ & $.23^{*}$ & .08 & $.24^{*}$ & $.27^{\star}$ & $.30^{*}$ & .12 & $.45^{\star}$ & $.41^{*}$ & $.43^{\star}$ & $.53^{*}$ & $.41^{*}$ & $.49^{*}$ \\
\hline
\end{tabular}

Nota: ESP = Esperanza; PRO = Proactividad; FIL = Filosofía de trabajo; EMP =Empatía; INN = Innovación; AUT = Autonomía; RIESG = Toma de riesgos; IND = Valores individuales; $\mathrm{COL}=$ Valores colectivos; $\mathrm{MIXT}=$ Valores mixtos; $\mathrm{ACAD}=$ Autoconcepto académico; $\mathrm{SOC}=$ Autoconcepto social; FAM = Autoconcepto familiar; FíSIC = Autoconcepto físico; ${ }^{*} p<.05$.

\section{Discusión}

El objetivo de la presente investigación es conocer algunos de los factores protectores frente a la violencia escolar, violencia que, en el caso de la República Dominicana, es una problemática recurrente, afectando a un $21.8 \%$ de la población (Román y Murillo, 2011). Para ello, se puso a prueba un modelo de ecuaciones estructurales en el que se incluyeron como factores protectores cuatro dimensiones del autoconcepto (académico, social, familiar y físico), la esperanza disposicional, los valores (tanto los individualistas, como los colectivistas y los mixtos) y las actitudes emprendedoras (proactividad, filosofía o ética profesional, empatía, innovación, autonomía y toma de riesgos). 
En primer lugar, y con respecto a la hipótesis que predecía que las dimensiones del autoconcepto pudiesen desempeñar un papel protector de las conductas violentas, los resultados mostraron evidencia, tanto en sentido afirmativo como negativo, en función de la dimensión concreta bajo estudio. Mientras que el autoconcepto familiar predijo negativamente las conductas violentas de los estudiantes, de forma que a mayor nivel de autoconcepto familiar, menor nivel de violencia, el autoconcepto social se relacionó de forma positiva, mostrando que aquellos estudiantes con mayor autoconcepto social eran también los más violentos. En cuanto al autoconcepto académico y físico, no resultaron ser predictores estadísticamente significativos. Estos resultados indican que, aunque de manera general la autoestima es considerada un factor protector ante problemas emocionales y conductuales, como es el caso de las conductas violentas, las cuales asocian el comportamiento agresivo de los adolescentes con una autoestima más baja, se recomienda ser cautos y no caer en generalizaciones, prestando atención a matices según las dimensiones. Por tanto, estos resultados siguen la línea de aquellas investigaciones que tienen una visión multidimensional de la variable autoconcepto.

También respaldan la importancia y sentido de la relación entre autoconcepto familiar y violencia, pues como Moreno et al. (2009) hallaran con las dimensiones del clima familiar, tanto cohesión como expresividad familiar, mostraron relaciones negativas con la violencia pura, la violencia manifiesta y la violencia instrumental. Al respecto añadir, como subrayan Martínez et. al, (2008), que existen estudios que señalan cómo los adolescentes violentos valoran de un modo negativo el ámbito familiar, informando de relaciones hostiles con sus padres (frecuentes conflictos, problemas de comunicación y conductas desafiantes en sus relaciones familiares) y perciben un bajo apoyo parental, variables que conforman una menor autoestima familiar (Dekovic et al., 2004; Morales y Costa, 2001; Musitu et al., 2001).
En cuanto a la relación entre autoconcepto social y conductas violentas, tal y como señala Estévez (2005), recientes estudios afirman que los adolescentes con problemas de agresividad en la escuela obtienen puntuaciones elevadas de autoconcepto social (Andreou, 2000; O'Moore y Kirkhan, 2001). Una posible explicación de estos resultados es que los adolescentes agresivos son en numerosas ocasiones figuras importantes en su grupo de iguales (GilfordSmith y Brownell, 2003; Hawley y Vaughn, 2003) e incluso populares y queridos entre sus compañeros (Salmivalli, 1998), lo que les hace disfrutar de ciertos beneficios sociales que se reflejan en esta dimensión de la autoestima (Little et al., 2003). Referente a la falta de relación entre autoconcepto académico y físico y conductas violentas, los resultados aportados en el presente trabajo son acordes con investigaciones previas, que mostraron una falta de relación entre ambos constructos (ver, por ejemplo, Cava et al., 2006).

En cuanto al papel de la esperanza disposicional, y tal y como se esperaba, esta se mostró como un protector frente a las conductas violentas en la escuela. Los resultados del modelo apuntaron una relación negativa y elevada entre ambas variables, siendo además el predictor con mayor capacidad explicativa. De esta forma, los tres componentes de la esperanza: la conceptualización de las metas, el desarrollo de estrategias para conseguir dichas metas y la motivación para utilizar dichas estrategias (Lopez et al., 2000; Snyder, 2000; Snyder et al., 2003) serían factores claves en la prevención de la violencia en los centros escolares.

Tal y como ocurría con el autoconcepto, las actitudes emprendedoras funcionan como protectores o no de la violencia en función de la dimensión que se estudie. Los resultados de esta investigación apuntan que variables como la empatía y la toma de riesgo se relacionan negativamente con las conductas violentas en la escuela, mientras que la filosofía o ética profesional lo hace de forma positiva. La empatía ha sido identificada previamente como un factor protector de la violencia escolar, dado que niveles escasos de empatía suelen conllevar 
BÚSQUEDA - Julio / Diciembre de 2015 - No. 15 (18 - 29)

conductas sociales incívicas, incluyendo las conductas violentas entre iguales (Díaz-Aguado, 2006; Martorell, González, Rasal y Estelles, 2009; Stassen, 2007).

El autoconcepto social como percepción que cada cual tiene de sus habilidades sociales, ser popular y mantener buenas relaciones con los otros, parece relevante y según Barón (2000) favorece la construcción de actitudes emprendedoras. Así, el relacionarse con los demás de forma adecuada ejerce efectos beneficiosos diferentes en contextos más allá del ajuste social, como por ejemplo, obtener un respaldo económico o fidelizar nuevos clientes, e incluso, se considera predictora de un mayor éxito en los negocios (Barón y Markman, 1999, citado en Barón 2000). Concretamente en el campo de las iniciativas emprendedoras, se sabe que estas no solo son productos de construcción individuales, sino que varias veces responden al esfuerzo grupal por lo que valores y creencias sobre sí mismo, sostenidas en el contexto de las interrelaciones sociales parecen ser favorecedoras de este tipo de iniciativas (Davidsson y Honing, 2003; Liñán y Santos 2007, citado en Liñán, 2008).

Finalmente, en lo que respecta a los valores personales, los valores mixtos son aquellos que se han mostrado protectores frente a las conductas violentas, mientras que los valores colectivos se han relacionado positivamente. Los valores individuales, por su parte, no mostraron relación. Aunque no existen estudios previos a este respecto, la hipótesis inicial era que todos los valores serían protectores frente a las conductas violentas. Los resultados contraintuitivos conseguidos deberán servir para que futuras investigaciones ahonden en la relación entre valores y conductas violentas en la escuela, para desentrañar si se trata de una particularidad de la muestra de estudio, o esta relación se da también en otros contextos, y buscar las posibles causas.

En síntesis, encontramos que los resultados del presente estudio ponen de manifiesto la existencia de factores protectores frente a las conductas violentas entre adolescentes dominicanos. Estos datos son observables, puesto que un conjunto de variables enmarcadas en la psicología positiva: autoconcepto familiar, esperanza disposicional, empatía, toma de riesgos y valores mixtos (a la vez individuales y colectivos), se encuentran asociadas de forma negativa con la conducta violenta. En este sentido, y siendo conscientes de las limitaciones de un estudio de corte transversal con medida en un único momento temporal, en función de la capacidad explicativa de estos protectores, cabe esperar que fomentándolos se consiga disminuir la conducta violenta entre adolescentes. Johannisson, autoridad en el campo del emprendimiento social, menciona que el espíritu empresarial requiere de la capacidad de organizar creativamente los recursos humanos, por lo que competencias como la empatía, atender al otro como si fuera uno mismo, formarían parte del autoconcepto social (Johannisson, 2011).

En esta línea, se concluye que los adolescentes con una elevada autoestima o autoconcepto familiar tienden a mostrar una menor implicación en este tipo de comportamientos agresivos (Cava et al., 2006; Estévez, Martínez y Musitu, 2006; O'Moore y Kirkham, 2001). De este modo, múltiples investigaciones relacionan los estilos educativos que usan madres y padres, con problemas de conducta de sus hijos: uso de alcohol y drogas, delincuencia, violencia, o abandono escolar (Ricardo, Mateu y Oliver, 2014). Es por ello que una estrategia complementaria al fomentar las variables protectoras mencionadas, requeriría de una enseñanza dirigida a padres que inculquen correctos estilos educativos. Pero la capacidad de actuar no se limita a estas variables del ámbito familiar, cada vez son más los estudios que se suman a la importancia de promover una actitud emprendedora dentro de las aulas, así como desarrollar un programa educativo en el que ocuparse de los componentes de la esperanza disposicional de los alumnos (conceptualización de las metas, desarrollo de estrategias para conseguir dichas metas y motivación para utilizar dichas estrategias). Con estas medidas se pretende inducir un cambio emocional y conductual que repercuta de forma positiva sobre la prevención y eliminación de las conductas violentas. 
Adicionalmente, organismos como la UNICEF atendiendo el llamado de gobiernos nacionales, organismos multilaterales, organizaciones no gubernamentales (ONG) y proveedores de servicios financieros hace un llamamiento a la necesidad de una educación financiera, social y sobre los medios para ganarse la vida, entre los que estaría autoemplearse, ya desde la infancia (UNICEF, 2013). Esta educación al tiempo podría servir para prevenir o atenuar la violencia en jóvenes. Efectivamente, desde un enfoque basado en los Derechos Humanos de cada niño o joven, promover la cohesión social es perfectamente compatible con una sociedad que potencie el emprendimiento. En concreto, la propia UNICEF, desarrolla iniciativas de apoyo psicosocial, aprendizaje social y emocional, reducción de riesgos y promoción de salud que podrían enlazarse con acciones de emprendimiento. Para que este tipo de acciones sean lo más eficientes posibles, es necesario seguir avanzando con la investigación en este campo.

\section{Referencias bibliográficas}

Andreou, E. (2000). Bully/Victim problems and their association with psychological constructs in 8-to 12-year old Greek schoolchildren. Aggressive Behavior, 26, 49-56.

Bustos, V., Oliver, A., y Galiana, L. (2015) Validación del Autoconcepto Forma 5 en universitarios de Perú: Una herramienta para la Psicología Positiva. Psicologia, Reflexao e Critica, 28, 690-697. DOI: 10.1590/1678-7153.201528406

Cava, M.J., Musitu, G., y Murgui, S. (2006). Familia y violencia escolar: el rol mediador de la autoestima y la actitud hacia la autoridad institucional. Psicothema, 18(3), 367-373.

Dekovic, M., Wissink, I. B. y Meijer, A. M. (2004). The role of family and peer relations in adolescent antisocial behaviour: comparison of four ethnic groups. Journal of Adolescence, 27, 497-514.

Díaz-Aguado, M. J. (2006). Sexismo, violencia de género y acoso escolar. Propuestas para una prevención integral de la violencia. Estudios de Juventud, 73, 38-58.

Estévez, E. (2005). Violencia, victimización y rechazo escolar en la adolescencia. (Tesis doctoral). Departamento de Psicología Social. Universitat de València.

Estévez, E., Martínez, B., Herrero, J., y Musitu, G. (2006). Aggressive and non-aggressive rejected students: An analysis of their differences. Psychology in the Schools, 43, 387-400.

Estévez, E., Martínez, B., y Musitu, G. (2006). La autoestima en adolescentes agresores y víctimas en la escuela: La perspectiva multidimensional. Intervención Psicosocial, 15, 223-232.

Francis, L. J., Robbins, M., y White, A. (2003). Correlation between religion and happiness: A replication. Psychological Reports, 92, 51-52.

Galiana, G., Oliver, A., Sancho, P., y Tomás, J. M. (2015). Dimensionality and Validation of the Dispositional Hope Scale in a Spanish Sample. Social Indicators Research, 120, 297-308.

García, F., y Musitu, G. (1999). Autoconcepto Forma 5. Madrid: TEA. Gifford-Smith, M. E. y Brownell, C. A. (2003). Childhood peer relationships: social acceptance, friendships, and social network. Journal of School Psychology, 41, 235-284.

Hawley, P. y Vaughn, B. (2003). Aggression and adaptation: The bright side to bad behavior. Introduction to special volume. Merrill-Palmer Quarterly, 49, 239-244.

Hu, L. T., y Bentler, P. M. (1999). Cutoff criteria for fit indexes in covariance structure analysis: Conventional criteria versus new alternatives. Structural Equation Modeling, 6, 1-55.

Johannisson, B. (2011). Sobre el carácter social del espíritu empresarial. En R. Varela (ed.), Desarrollo, innovación y cultura empresarial (pp. 131-157). Santiago 
BÚSQUEDA - Julio / Diciembre de 2015 - No. 15 (18 - 29)

de Cali: Universidad Iceci-Centro del desarrollo del Espíritu Empresarial.

Kirby, D. (2004). Entrepreneurship Education: ¿Can Schools Meet the Challenge? Journal Education and Training, 46, 510-519.

Kline, R. B. (2011). Principles and Practice of Structural Equation Modeling ( $3^{\mathrm{a}} \mathrm{Ed}$.). New York, NY: Guilford Press.

Landwerlin, G. M. (2011). Individualización y solidaridad familiar. Barcelona: Fundación "La Caixa".

Little, T., Brauner, J., Jones, S., Nock, M. y Hawley, P. (2003). Rethinking aggression: A typological examination of the functions of aggression. Merrill-Palmer Quarterly, 49, 343-369.

Lopez, S. J., Ciarlelli, R., Coffman, L., Stone, M., y Wyatt, L. (2000). Diagnosing for Strengths: On Measuring Hope Building Blocks. In C. R. Snyder (Ed.), Handbook of hope: Theory, measures and applications (pp. 57-85). San Diego, CA: Academic Press.

Marques, S. C., Lopez, S. J., y Mitchell, J. (2013). The role of hope, spirituality and religious practice in adolescents' life satisfaction: Longitudinal findings. Journal of Happiness Studies, 13, 251-261.

Martínez, B., Murgui, S., Musitu, G. y Monreal M.C. (2008). El rol del apoyo parental, las actitudes hacia la escuela y la autoestima en la violencia escolar en la adolescencia. Internacional Journal of Clinical and Health Psycohology, 8(3), 679-692.

Martínez, M.; Buelga, S., y Cava, M. J. (2007). La satisfacción con la vida en la adolescencia y su relación con la autoestima y el ajuste escolar. Anuario de Psicología, 38(2), 5-15.

Martorell, C., González, R., Rasal, P., y Estellés, R. (2009). Convivencia e inteligencia emocional en niños en edad escolar. European Journal of Education and Psychology, 2, 69-78.
Morales, J. M. y Costa, M. C. (2001). La prevención de la violencia en la infancia y la adolescencia. Una aproximación conceptual integral. Intervención Psicosocial, 10(2), 221-239.

Moreno, D., Estévez, E., Murgui, S., y Musitu, G. (2009). Relación entre el clima familiar y el clima escolar: El rol de la empatía, la actitud hacia la autoridad y la conducta violenta en la adolescencia. International Journal of Psychology and Psychological Therapy, 9(1), 123-136.

Moreno, D., Estévez, E., Murgui, S., y Musitu, G. (2009). Reputación social y violencia relacional en adolescentes: el rol de la soledad, la autoestima y la satisfacción vital. Psicothema, 21(4), 537-542.

Moreno, D., Ramos, M. J., Martínez, B., y Musitu, G. (2010). Agresión manifiesta y ajuste psicosocial en la adolescencia. SUMMA Psicológica UST, 7(2), 45-54.

Morgan, J., y Farsides, T. (2009). Measuring meaning in life. Journal of Happiness Studies, 10, 197-214.

Musitu, G., Buelga, S., Lila, M. S. y Cava, M. J. (2001). Familia y Adolescencia. Madrid: Síntesis.

Musitu, G., Estévez, E., y Emler, N. (2007). Adjustment problems in the family and school contexts, attitude towards authority and violent behaviour at school in adolescence. Adolescence, 42, 779-794.

O'Moore, M. y Kirkham, C. (2001). Self-esteem and its relationship to bullying behavior. Aggressive behavior, 27, 269-283.

Oliver, A., y Galiana, L. (2015). Development and validation of the Entrepreneurial Attitudes Scale for Students (EASS). The Spanish Journal of Psychology, 18, E4.

OMS (2002). Informe Mundial sobre Violencia y la Salud. Washington D.C: Publicación Organización Mundial de la salud. Recuperado de: http://www.who.int/ 
violence_injury_prevention/violence/world_ report/en/summary_es.pdf.

Quintanilla, A., Montgomery, W., y Malaver, C. (2009). Modos de afrotamiento y conducta resiliente en adolescentes espectadores de violencia entre pares. Revista IIPSI, 12(1), 153-171.

Ricardo, G., Mateu, C. y Oliver, A. (2014). Habla con tus hijos, no a tus hijos: intervención en parenting empático. I Jornada Internacional de Servicios Sociales. Valencia, España.

Román, M., y Murillo, F. J. (2011). América latina: violencia entre estudiantes y desempeño escolar. Revista de CEPAL, 104, 37-54.

Salmivalli, C. (1998). Intelligent, attractive, wellbehaving, unhappy: the structure of adolescents' self-concept and its relations to their social behavior. Journal of Research on Adolescence, 8, 333-354.

Sánchez, J.C.; Gutiérrez, A.; Carballo, T.; Quintana, R. y Caggiano, V. (2010). El emprendedor desde el enfoque de la psicología positiva. International Journal of Developmental and Educational Psychology/INFAD Revista de Psicología, 36, 35-42.

Schwartz, S. H. y Bilsky, W. (1990). Toward a theory of the universal content and structure of values: Extensions and cross cultural replications. Journal of Personality and Social Psychology, 58, 878-891.

Serrano. Á., y Iborra, I. (2005). Violencia entre compañeros en la escuela. Valencia: Centro Reina Sofia para el Estudio de la Violencia.

Snyder, C. R. (2000). Handbook of hope: Theory, measures and applications. San Diego, CA: Academic Press.

Snyder, C. R., Harris, C., Anderson, J. R., Holleran, S. A., Irving, L. M., Sigmon, S. T., Yoshinobu, L., Gibb, J., Langelle, C., y Harney, P. (1991). The will and the ways: Development and validation of an individual-differences measure of hope. Journal of Personality and Social Psychology, 60, 570-585.
Snyder, C. R., Lopez, S. J., Shorey, H. S., Rand, K. L., y Feldman, D. B. (2003). Hope Theory, Measurements, and Applications to School Psychology. School Psychology Quarterly, 18(2), 122-139.

Soledad, M., Hopkins, C., y Mirna, B. (2007). Directorio de Expresiones Organizativas de Mujeres a Nivel Municipal 2007. El Salvador: FUNDE, Fundación Nacional para el Desarrollo.

Stassen, K. (2007). Update on bullying at school: science forgotten? Developmental Review, 27, 90-126.

Tanaka, J. S. (1993). Multifaceted conceptions of fit in structural equation models. En K. A. Bollen y J. S. Long, Testing Structural Equation Models (pp. 10-39). Newbury Park, CA: Sage.

UNICEF (2013) Educación social y financiera para la infancia. ESCUELAS AMIGAS DE LA INFANCIA. Recuperado de: http:// www.unicef.org/cfs/files/CFS_FinEd_Sp_ Web_8_5_13

Vargas, Y. (2014). Estudio de Prevalencia, Tipología y Causas de la Violencia en los Centros Educativos de Básica y Media de la República Dominicana. Santo Domingo: Ministerio de Educación (MINERD), Instituto Dominicano de Evaluación e Investigación de la Calidad Educativa (IDEICE) y Universidad Iberoamericana (UNIBE).

Velasco, M. J. (2013). Violencia reactiva e instrumental. La impulsividad como aspecto diferenciador. Revista de educación, 361, 665-685.

Vesper, K. H. (1990). New Venture Strategies. University of Illinois at UrbanaChampaign's Academy for Entrepreneurial Leadership Historical Research Reference in Entrepreneurship. Recuperado el 20 de octubre de: http://ssrn.com/ abstract $=1496217$. 